\title{
Técnica original de perineorrafía post-episiotomía
}

\author{
Doctor Miguel A. Fernández Bastidas \\ (Trabajo presentado al concurso anual abiertu por la Sociedad \\ Colombiana de Obstetricia y Ginecología).
}

El presente estudio, hecho sobre ciento treinta $\mathrm{y}$ cinco historias de episiotomías practicadas personalmente por nosotros en la Clínica Primero de Mayo de Bogotá, sobre un totái de ciento ochenta y seis partos atendidos entre el mes de febrero de 1952 y agosto del 53, tiene por objeto, además de analizar las indicaciones que nos movieron a ejecutarlas en el segundo período del parto, informar sobre los resultados posteriores obteridos con las suturas practicadas según técnica original.

De una gran popularidad en los últimos años en la práctica obstétrica la episiotomía se ha generalizado, al decir de Eastman (1) por tres razones fundamentales. Primera, por sustituir la laceración contusa que con mucha frecuencia se presenta en el segundo período del parto, por un corte neto quirúrgico que da una incisión fácil de reparar y de mejor cicatrización cue un desgarro. Segunda: por evitarle a la cabeza fetal servir de "dilatador" contra una obstrucción perineal, con cuya presión puede dar lugar a una irreparable lesión cerebral. Tercera: por acortar la duración del segundo período del parto, evitando la compresión exagerada de las partes blandas por el polo cefálico.

Conocida suficientemente por el especialista la anatomia perineal femenina y admitido por varios autores (2-3) el importante papel ásumido por este piso músculo-aponeurótico como medio de sostén uterino, nos parece necesario agregar a las ventajas ya enumeradas la de lograr la integridad funcional del periné al ser ejecutada la episiotomía en el momento oportuno. 
La episiotomia puede hacerse siguiendo el rafé ano-vulvar sobre la línea media o episiotomía mediana; o bien con un corte medio-lateral derecho o izquierdo, iniciado en la parte media de la horquilla vulvar y continuado a uno de los lados del eśfinter anal, caso en el cual se le denomina lógicamente episiotomía medio-lateral. Para nuestro estudio, empleamos únicamente la incisión medio-lateral derecha en todos los casos; y la hallamos ventajosa, por no presentar el peligro de complicación con un desgarro de tercer grado por prolongación hacia el recto.

La mediana tiene muchos partidarios, quienes, al considerarla más anatómica, aducen ia ventaja de su más fácil sutura. Los que la practican sistemáticamente en el extranjero, no solamente no temen a la complicación del desgarro de tercer grado, sino que en casos presenciados por nosotros en un hospital rorteamericano (no sabemos si hay publicaciones al respecto), antes de que se produjera éste, y ante la desventaja de entrar más tarde a suturar un esfínter desgarrado hacían la incisión quirúrgica de él. Claro está que a nuestro modo de ver, semejante conducta no deja de tener sus graves inconvenientes. Por esta y otras razones preferimos ejecutar la segunda modalidad, en la cual, como lo veremos más adelante, teniendo un poco de cuidado se logra un reparo perfectamente satisfactorio.

Pensar que un periné no desgarrado pero sí relajado al máximo después de "maniobras de protección perineal", constituye un éxito en la atención de un parto, es no soiamente ilógico sino, tomado como rutina, altamente perjudicial, tanto para la madre como para el feto; a la primera se le predispone a un prolapso genital más o menos precoz, y al segundo se le expone a una lesión cerebral por aumento de presión intracraneana, que si no le es fatal le puede convertir en un demente incorregible.

Greenhill (4) da las siguientes indicaciones absolutas para su práctica: primera: la existencia de un periné resistente; segunda: las causas que demoran la salida de la cabeza a través de la vulva, tales como cicatrices de antiguos desgarros (caso en el cual se debe completar con una colpoperineorrafia), feto grande, mecanismo anormal expulsivo o desproporción mecánica en ei segundo perfodo. Tercera: el parto de un feto premoturo. Cuarta: la urgencia de una extracción rápida por sufrimiento fotal. Quinta: el peligro de un posible desgarro del esfínter en el momonto en que el polo fetal franquea la horquilla vulvar.

Siguiendo estas indicaciones vamos a analizar los casos estudiados. 


\section{CUADRO NUMERO 1}

\section{Episiotomías según las indicaciones}

a) por cicatrices de antiguos desgarros ..... 5 casos

b) por feto grande $\ldots \ldots \ldots \ldots$

c) por mecanismo expulsivoanormal $\ldots \ldots c c 27$

d) por periné resistente . . . . . . . . . . . . . . . . 22

e) por parto de feto prematuro ... . . . . . . 18

f) por sufrimiento fetal (extracción con forceps) . . . . . . . . . . . . . . . . . . . . . 6 6

g) peligro de desgarro de tercer grado . . . . 20 "

h) por primiparidad (algunas extracciones con forceps) . . . . . . . . . . . . . . . . . . . . . . 28 ",

Total de casos . . . . . . . . . . . . . . . $\quad .355$

\section{CUADRO NUMERO 2}

Episiotomías en relación con la paridad y madurez fetal I-Primigestantes (44 casos o $32,5 \%$ ).

a) con fetos de peso inferior a 2.500 grs. . . . 12 casos

b) con fetos de más de 2.500 grs. . . . . . . 32 " II-Multigestantes ( 91 casos o 67,5\%).

a) con fetos de menos de 2.500 grs. . . . . . . 11 casos

b) con fetos de peso superior a 2.500 grs. . . . 80 "

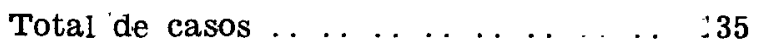

\section{CUADRO NUMERO 3}

Episiotomias de acuerdo con presentaciones $y$ variedades de posición

I-Vértice:

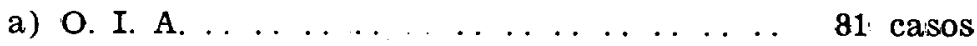

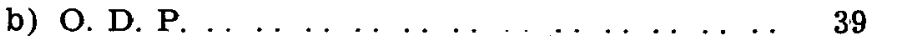

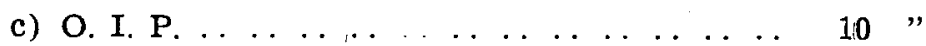

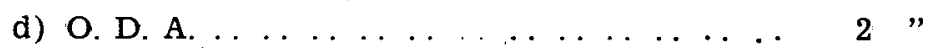

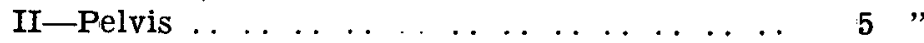

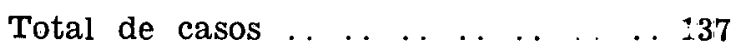

Nota: el total de 137 se explica por haber 2 casos de parto gemelar; uno con O. I. A. y S I. I.; y otro con ambos fetos en O. I. A. Como se puede apreciar (cuadro número 1), 28 casos corres- 
ponden a episiotomías practicadas en primigestantes como indicación principal, en las cuales la resistencia exagerada de los planos musculares perineales retardó notablemente al segundo período del parto. Así, pues, siguiendo a Harr y Buckman (5), quienes dan la primiparidad como la causa predisponente más frecuente de muerte fetal en eì segundo período; no dudamos en recomendar se practique la episiotomia sistemáticamente en todo parto en primípara. En el cuadro número 1 no figuran todas las primegestantes episiotomizadas dentro de la casilla correspondiente a esta indicación, por haberse hecho muchas más con la indicación de feto prematuro que con la de parto en primipara (Ver cuadro número 2).

Además del parto prematuro y la primiparidad, un mecanismo expulsivo anormal por variedades posteriores a por distocias dinámicas juzgables por la duración del trabajo; un periné resistente cicatricial o no, el peligro de un desgarro do tercer grado en partos de multiparas con fetos grandes, son más de otras indicaciones menos frecuentes, las causas que nos impulsaron a practicar la intervención, y que también aparecen suficientemente explicadas en los cuadros números 1,2 y 3 .

En relación con el parto en pelvis, la opinión actial es: que se le debe practicar a la paciente una episiotomía ampia (una y media veces mayor que en la de parto en cefálica) (6) con mira a facilitar la extracción de la cabeza última, y conjurar un desgarro de tercer grado, al presentarse un diámetro cefálico demasiado grande; y que su práctica precoz facilita igualmente la ejecución de maniobras manuales, tales como ia extracción podálica (en pelvis incompleta) o el "Mauriceau" en cabeza última, acelerando al facilitarla, la evolución final del parto (7) y contribuyendo a hacerlo menos traumático para el feto.

Los poquísimos casos de presentación de pelvis que figuran en nuestra estadística, y su inclusión bajo la indicación "mecanismo expulsivo anormal" (cuadro número 1), explica por qué no figura como una de las principales indicaciones.

\section{Técnica de sutura}

Después de juzgar las indicaciones de una intervención, es de sana lógica considerar la técnica a seguir; y al describir ésta, abordar el motivo del presente trabajo. Para su mayor claridad, recomendamos observar las figuras adjuntas, en las cuales, a nuestro modo de ver, se da suficiente luz al respecto, ahorrándose una explicación que no llenaría su objeto (Figuras 1, 2, 3, 4). 


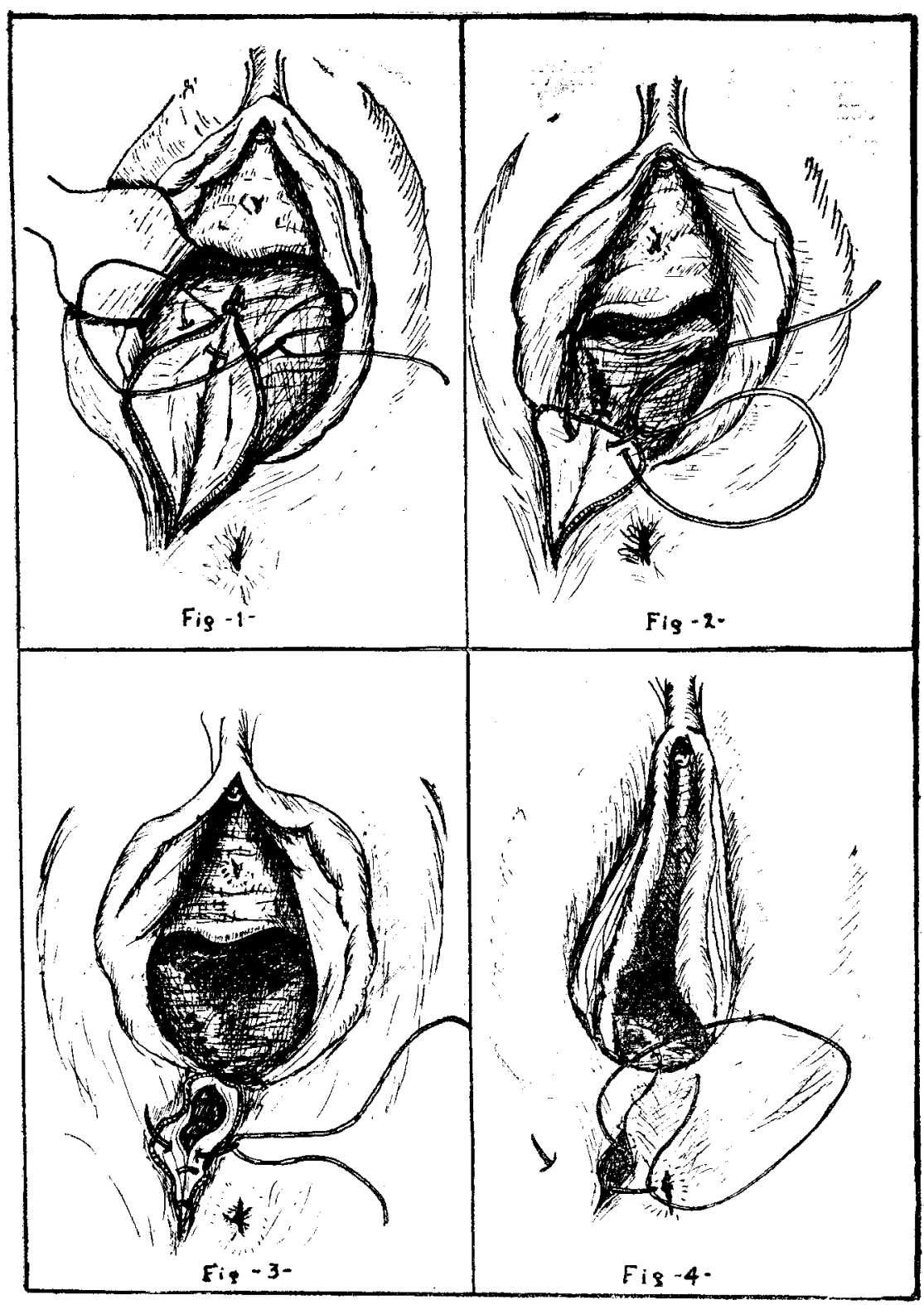

Fig. 1.-Primer tiempo; plano mucoso, con sutura continua festoneada. Fig. 2.-Terminación del plano mucoso, pasando el hilo al plano muscular profundo y anulando; queda así colocado el primer punto muscular, para continuar con puntos separados. Fig. 3.-Segundo tiempo: plano aponeurótico, iniciado de atrás a adelante. Fig. 4.-Tercer tiempo: Sutura in'radérmica; con el último punto sacado a distancia, $\mathrm{y} \sin$ anudar. 
Por no darle a esta intervención obstétrica la importancia que se merece, con frecuencia vemos hacer una pseudo-sutura de la mucosa y otra de la piel can puntos separados, generalmente con material de sutura inapropiado, que luego en el puerperio lógicamente da lugar a una mala cicatrización, tardía y rara vez anatómica; que como gran triunfo cura por "segunda intención" (8). (8).

En la técnica propuesta sólo usamos anestesia general por inhalación (ciclo 02, trilene), y no usamos la local pur parecernos poco práctica (9. Como instrumental: separadores, pinzas de Allis, pinzas de disección con y sin garra, porta-agujas largo, y un par de agujas curvas americanas, una de borde redondo para mucosa y músculo, y una cortante para la sutura intradérmica. Como material de sutura en todos los planos: catgut cromadc 0 ó 00.

Como se ve en las figuras, la sutura da una reconstrucción perfecta, no quedando ningún nudo superficial a nivel de la horquilla, que posteriormente, al dar lugar a la formacion de quistes o granulomas pequeños hagan consultar a la enferma por dispareunia. La forma como se termina la sutura intradérmica sin anudarla, pasando simplemente el cabo: con la aguja a través del plano dérmico subyacente, da con lo señalado anteriormente la originalidad a esta sencilla técnica que ponemos en consideración de los especialistas, para que sea practicada.

En todos los casos suturados la cicatrización fue perfecta, logrando el levantamiento precoz de la paciente al tercero o cuarto día del puerperio. Como único inconveniente anotamos el molesto dolor perineal al tratar de sentarse precozmente la paciente, dolor que solamente en dos casos hizo necesaria la aplicación de una infiltración local anestésica, y que desapareció por completo al sexto día. Igualmente debemos anotar un caso aue no figura en nuestra estadística: Una paciente a quien se le practicó la sutura propuesta después de ocho días de puerperio, y con el fin de restaurar una sutura abierta suturada por otra técnica; se logró después de avivar bordes, una cicatrización perfecta al cuarto día.

En cuanto a la cicatriz dejada, se observaron varias enfermas episiotomizadas anteriormente al ser atendidas en partos subsiguientes y se pudo observar que el periné aparecía elástico e intacto, con toda la apariencia del de una primigestante; a estas pacientes se les practicó una nueva episiotomia, no por periné resistente cicatricial, sino para evitar que por omisión se hubiera perdido lo logrado en el parto anterior. 
La técnica propuesta tiene sobre las demás las siguientes ventajas: sobre la llamada sutura en "8", en que al no usar material inabsorbible, éste no requiere ser retirado más tarde, evitando además someter a la paciente al incomodísimo sistema de pinzas e hilos anudados, después de ser sacada de la sala. Sobre el sistema de suturar la piel con puntos separados perforante de "Corachan" tiene la ventaja de que al no perforar la piel, y al evitar la tensión de ésta, nunca se presenta la complicación de aue el hilo la desgarre al edematizarse. Ahora bien: sobre la tan frecuente apl1cación de ganchos o agrafes, después de una sutura muscular poco minuciosa, la ventaja es mayor aún, pues le evita a la paciente la molesta quitada de los ganchos y sobre todo el peligro de infección, por la dificultad de practicar un minucioso aseo diario perineal, por el dolor agudo que se despierta en la enferma al practicarlo.

\section{Resumen}

Se presentan ciento treinta y cinco casos de episiotomía medio-lateral derecha, y sus indicaciones que la motivaron. Se describe además una técnica original de sutura, que logra reconstruir los planos perineales, con las ventajas: I-No usar material inabsorbible que requiera ser retirado más tarde, y II-que' este material al terminar la sutura quede perfectamente incluído en los tejidos, sin peligro de ocasionar más tarde granuiaciones o pequeños quistes que puedan dar lugar a ia aparición de dispareunia. En todos los casos presentados el proceso de cicatrización fue óptimo y sin complicaciones.

\section{BIBLIOGRAFIA}

(1) Eastman. William.- “Obstetrics". 10 th Edit. Appleton: 410. 1950.

(2) Meigs J. E.-Sturgis S. H. Progress Gynecology. Vol. II. Grune S. 802.

(3) Phaneuf Louis E.-Progresos de la ginecología. Vol. II. Ed. Científico Médica. Barcelona, Madrid, Valencia. 1953. 768-770.

(4) Greenhill.-De Lee Principles and Practice of Obstetrics. Tenth Edit. Nosby St. Louis. L. 951: 249.

(5) Harr James A. Bukman Myron I. Am. J. Obst and Gynec. 61: 348. 355. Feb. 51.

(6) Barnes C.-(Ohio State Univ.) Potgrad. Med. 12; 121-126. Aug. 1952. (Year Book. 1953).

(7) Dorr Edward M. (Norwestern Univ.) S. Clin North America. 33: 77 . 85. Feb. 53.

(8) Wallis Henry (Caracas)-Rev. Obst. y Gynec. Caracas. 12: 216. 1952.

(9) Weber Fetchco and Carrol. Am. J. Obst and Gynec 64: 196. 1952.

(10) Greenhill.-De Lee Principles and Practica of Obstetrics Tenth Edit. Mosby St. Louis. 1951. 